\title{
lodine monitoring models contribute to avoid adverse birth outcomes related more than adequate iodine intake
}

Jinju Dong ${ }^{1+}$, Shouyan Liu ${ }^{2+}$, Lingyun Wang ${ }^{3}$, Xingjian Zhou', Qinghong Zhou', Congli Liu', Jingrui Zhu Weilan Yuan ${ }^{3}$, Wang-yang $\mathrm{Xu}^{4^{*}}$ and Jie Deng ${ }^{1 *}$

\begin{abstract}
Background: lodine plays an important role in pregnancy. How to maintain adequate iodine intake amongst pregnant women in each trimester of pregnancy to prevent adverse birth outcomes in central China is a challenge for clinical practice.

Methods: 870 pregnant women and their infants were enrolled in the study. Urinary iodine concentration (UIC) was measured using an inductively coupled plasma mass spectrometry (ICP-MS). Maternal and newborn information were obtained during follow-up. Multinomial logistic regression models were established.

Results: Median UIC of pregnant women was $172 \pm 135 \mu \mathrm{g} / \mathrm{L}$ which is currently considered to be sufficient. Multivitamin supplements containing iodine, iodized salt intake and frequent milk intake were significantly associated with higher UIC. Multivariate logistic regression analysis showed that multivitamin supplements containing iodine and milk consumption were risk factors for more than adequate iodine (UIC $\geq 250 \mu \mathrm{g} / \mathrm{L}$ ). lodinerich diet was significantly related to heavier birthweight, larger head circumference and longer femur length of the newborns while more than adequate iodine intake (UIC $\geq 250 \mu \mathrm{g} / \mathrm{L})$ was a risk factor for macrosomia. Logistic regression models based on potential risk factors involving iodine containing supplements and iodine-rich diet were established to predict and screen pregnant women with high risk of more than adequate iodine intake among local pregnant women in different trimesters and guide them to supplement iodine reasonably to prevent the risk.

Conclusions: Multivitamin supplements containing iodine and milk consumption were risk factors for maternal UIC $\geq 250 \mu \mathrm{g} / \mathrm{L}$ which was a risk factor for macrosomia. lodine monitoring models were established to provide guidance for pregnant women to reduce the risk of more than adequate iodine intake, thereby contributing to reduce the risk of having a macrosomia.
\end{abstract}

Keywords: More than adequate iodine, Monitoring model, Macrosomia

*Correspondence: Rita_xwy@126.com; gelanxiu123@163.com

†Jinju Dong and Shouyan Liu contributed equally to this work. ${ }^{4}$ Singlera Genomics (Shanghai) Ltd, Shanghai 201318, China

'Department of Gynaecology and Obstetrics, Xiangyang No.1 People's Hospital, Hubei University of Medcine, Xiangyang 441000, China

Full list of author information is available at the end of the article

\section{Introduction}

Iodine is an essential component for the synthesis of thyroid hormones (THs) which are important for the growth and maturation of fetus and the development of brain [1]. Sufficient iodine intake and TH levels are essential to prevent the birth abnormality of the offspring $[2,3]$. Previous studies have shown that the increased

(c) The Author(s). 2021 Open Access This article is licensed under a Creative Commons Attribution 4.0 International License, which permits use, sharing, adaptation, distribution and reproduction in any medium or format, as long as you give appropriate credit to the original author(s) and the source, provide a link to the Creative Commons licence, and indicate if changes were made. The images or other third party material in this article are included in the article's Creative Commons licence, unless indicated otherwise in a credit line to the material. If material is not included in the article's Creative Commons licence and your intended use is not permitted by statutory regulation or exceeds the permitted use, you will need to obtain permission directly from the copyright holder. To view a copy of this licence, visit http://creativecommons.org/licenses/by/4.0/ The Creative Commons Public Domain Dedication waiver (http://creativecommons.org/publicdomain/zero/1.0/) applies to the data made available in this article, unless otherwise stated in a credit line to the data. 
risk of abortus, stillbirth, low birthweight infants, macrosomia, preterm delivery, neurological damage, and intellectual impairment are closely related to maternal iodine deficiency [4-7]. Therefore, adequate iodine intake during pregnancy is an important measure to ensure the health of pregnant women and the growth and maturation of fetus.

More than $90 \%$ of the body's iodine sources are food, and most of the iodine is excreted through urine [8]. Therefore, UIC acts a good index to reflect the population iodine status during pregnancy [9]. Chinese government has implemented a universal salt iodization (USI) policy since 1996. In the beginning, the Ministry of Health stipulated that the standard of iodine content in edible salt was as follows: the iodine concentration of iodized salt (calculated by iodine ion) was processed to 50 $\mathrm{mg} / \mathrm{kg}$, not less than $40 \mathrm{mg} / \mathrm{kg}$ when it was manufactured, not less than $30 \mathrm{mg} / \mathrm{kg}$ when it was sold, and not less than $20 \mathrm{mg} / \mathrm{kg}$ when it was used by users. Adjusted continuously, in 2011, the Ministry of Health issued the "iodine content of edible salt", which stipulated that the average level of iodine content in edible salt should be lowered to $20 \mathrm{mg} / \mathrm{kg} \sim 30 \mathrm{mg} / \mathrm{kg}$, which can be divided into $20 \mathrm{mg} / \mathrm{kg}, 25 \mathrm{mg} / \mathrm{kg}$ and $30 \mathrm{mg} / \mathrm{kg}$. One or two kinds of iodized salt can be supplied according to the iodine nutrition level of people in the province. China has nearly wiped out iodine deficiency disease (IDD) in the past two decades. China is currently considered to be iodine sufficient [10]. Although iodine levels in many provinces have been greatly improved, the harm of iodine excess to pregnant women and fetus is also attracting attention. A study from Wuhan, another large city in Hubei Province, pointed out that maternal iodine deficiency and excess during pregnancy have adverse effects on fetal growth and development, and put forward the necessity of monitoring the iodine status of pregnant women to ensure normal iodine nutrition during pregnancy [11]. Research reports in Henan Province showed that UIC of reproductive-age women and pregnant women were more than adequate in recent years and women with UIC above $249 \mu \mathrm{g} / \mathrm{L}$ was significantly correlated with increased risk of abnormal pregnancy outcomes $[12,13]$.

The present study aimed to assess iodine status in central China and explore the effect of maternal UIC on neonatal outcomes. Because the best indicator to evaluate iodine status is still controversial, and considering the influence of iodized salt and iodine-rich diet on iodine status and reducing the influence of excessive iodine intake on newborns, we finally constructed a model in each trimester to predict and screen pregnant women with high risk of more than adequate iodine intake based on potential risk factors involving iodine containing supplements, iodized salt and iodine-rich diet and guided them to take iodine reasonably, thus helping to reduce the risk of macrosomia related more than adequate iodine intake.

\section{Methods \\ Participants}

In this study, 1023 pregnant women were recruited from the obstetrics department of the No.1 People's Hospital of Xiangyang City, Hubei Province and the No.1 People's Hospital of Pingdingshan City, Henan Province, from December 2017 to December 2019. The exclusion criteria were as follows: (1) a history of primary and metastatic tumor; (2) a history of severe cardiovascular and cerebrovascular disease, such as stroke, myocardial infarction, myocarditis, hereditary heart diseases and coronary heart disease; (3) a history of drug abuse; (4) a history of thyroidectomy; (5) infant mortality or major defects; (6) loss of follow-up; (7) lack of laboratory examination or questionnaire. Finally, 870 pregnant women and their newborns were included in the study. Participants filled in the clinical questionnaire including age, gestational weeks, pre-pregnancy body mass index (BMI), dietary habits, iodine supplement, use of iodized salt and medical records during pregnancy. Passed after three experts reviewed the clarity and understanding of the questionnaire and agreed to all items, data was collected by trained nurses via face-to-face interview. The construct validity and reliability of the questionnaire were analyzed. The Kaiser-Meyer-Olkin value (KMO) coefficient was 0.66. Bartlett's test yielded $P<0.0001$ representing the quality of the sample. The reliability coefficient (alpha) of the questionnaire was 0.85 , which was considered acceptable. Gestational weeks were divided into: First trimester, 1-12 weeks; Second trimester, 13-27 weeks; Third trimester, 28-40 weeks. BMI (kg/ $\mathrm{m}^{2}$ ) were divided into four subgroups including below 18.5, 18.5-23.9, 24-27.9, and above 28 according to China standard (National Health and Family Planning Commission of the People's Republic of China, 2013). Influence of diet, including seafood (three groups: no seafood consumption; occasional: 1-2 meal containing seafood per week on average; frequent: at least 3 meal containing seafood per week), egg (three groups: no egg consumption; occasional: 1-2 eggs per week on average; frequent: at least 3 eggs per week), cow' milk and yogurt (three groups: no consumption; occasional: $1-500 \mathrm{ml}$ per week on average; frequent: at least $500 \mathrm{ml}$ per week), were recorded according to the above classification. Xiangyang and Pingdingshan Municipal governments both chose iodized salt with $25 \mathrm{mg} / \mathrm{kg}$ iodine. Every pregnant woman was required to provide the package (including nutrition table) of household salt for cooking. The photo template of the package of household salt was shown in Supplementary Fig. 1. Pregnant women 
with iodized salt for cooking is defined as the use of iodized salt, without iodized salt for cooking is defined as no iodized salt use. The multivitamin tablets prescribed by the doctor for some pregnant women with vitamin deficiency contain $50 \mu \mathrm{g}$ iodine / per tablet. Pregnant women take one or two tablets daily $(50 \mu \mathrm{g}$ or $100 \mu \mathrm{g}$ of iodine intake) according to the doctor's advice. Whether each pregnant woman used multivitamin tablets, or if they bought them privately, and how much iodine the tablets contain were recorded. Pregnant women taking multivitamin supplements containing iodine are defined as iodine supplements, pregnant women taking multivitamin supplements without iodine or pregnant women not taking multivitamin supplements are defined as noniodine supplements. However, in this study, all participants received iodine supplements from multivitamin supplements and no pregnant women was given pure iodine supplements, so we descripted it as multivitamin containing iodine instead of iodine supplements. Newborn information including birth date, sex, length, weight, femur length and head circumference were obtained from medical records. The study has been performed in accordance with the Declaration of Helsinki and was approved by the Research Ethics Committee (201702170b) of Hubei Medical College. Informed consents were provided for all participants.

\section{Urine iodine measurement}

Participants were asked to void their bladders in the morning, then started a 24-h urine sampling, and $1.5 \mathrm{ml}$ of urine from a random urine was taken during the 24-h sampling process. Urine samples were placed at $4{ }^{\circ} \mathrm{C}$ and detected in duplicate by inductively coupled plasma mass spectrometry (ICP-MS) (Agilent Technologies, Inc., Tokyo, Japan) as described in a previous study within $8 \mathrm{~h}$ after receiving [14]. In short, $100 \mu \mathrm{l}$ urine samples were extracted with ammonium hydroxide solution. Tellurium was used as an internal standard. Selfmade quality control material was used in every run to ensure the accuracy of the method. The average concentration of the quality control sample is $140 \pm 4.5 \mu \mathrm{g} / \mathrm{L}$. Coefficient of variation (CV) was $3.19 \%$. Blank solution was routinely prepared and tested to monitor potential iodine cross contamination in urine samples. Participate in the External Quality Assessment (EQA) run by the National Health Commission of the People's Republic of China twice 1 year. A recent "Successful" participation certificate of EQA issued by the National Health Commission for the ICP/MS method in December 2020 was attached as Supplementary Fig. 2. The iodine status of pregnant women is graded according to the WHO/ ICCIDD reference, UIC $<150 \mu \mathrm{g} / \mathrm{L}$ is defined as deficiency, UIC of $150-250 \mu \mathrm{g} / \mathrm{L}$ is defined as adequate, UIC of $250-500 \mu \mathrm{g} / \mathrm{L}$ is defined as more than adequate and $\mathrm{UIC} \geq 500 \mu \mathrm{g} / \mathrm{L}$ is defined as excess [15].

\section{Multinomial logistic regression model}

In the machine learning framework, the logistic regression models of the score were calculated using the variables (clinical risk factors) to predict risk. The logistic score according to the logistic regression equation can be obtained via the following formula:

Predicted risk probability $=\frac{\mathrm{e}^{\left(\beta 0+\sum \beta^{\beta i x i)}\right.}}{1+\mathrm{e}^{(\beta 0+} \sum \beta_{\mathrm{ixi} i)}} \beta 0$, the constant of the logistic regression formula. Bi, the coefficient of the variable $X i$. Multinomial logistic regression is an extension of ordinary binary logistic regression analysis, in which classification dependent outcomes have more than two. The results were structured into three outcomes: iodine deficiency, adequate iodine and more than adequate iodine. Multinomial logistic regression models were selected to predict the probability of each outcome in different (three) trimester. The predictive values of outcomes were evaluated by the receiver operating characteristic (ROC) curve.

\section{Statistical analysis}

Statistical analyses were conducted by using Python software (Version 3.6) (https://www.python.org). Python is an interpretive and general-purpose computer programming language which was designed by Guido van Rossum in the late 1980s. It has a dynamic type of system and emphasizes readability and rapid prototyping. Python keeps the second position in the TIOBE index (https://www.tiobe.com/tiobe-index/). SciPy. stat subpackage in Python was used to data processing and statistics. Continuous variables were shown as mean/ median \pm standard deviation (SD), and categorical variables as frequency or percentage. The association between UIC and clinical characteristics, and the association between UIC and infant characteristics were analyzed by using ANOVA or Kruskal-Wallis $\mathrm{H}$ test. The Sklearn package in Python was applied to construct the model and calculate the area under the curve (AUC) of the ROC. $P<0.05$ was set as statistically difference.

\section{Results}

\section{lodine status among pregnant women in the Central China}

The median UIC was $172 \pm 135 \mu \mathrm{g} / \mathrm{L}$ among the pregnant women. About $30.5 \%$ of the pregnant women (265/ $870)$ were in the adequate UIC range $(150-249 \mu \mathrm{g} / \mathrm{L})$. Nearly $32.3 \%(281 / 870)$ showed a UIC of $250 \mu \mathrm{g} / \mathrm{L}$ or greater. On the contrary, $37.2 \%$ (324/870) of participants had a UIC $<150 \mu \mathrm{g} / \mathrm{L}$ (Supplementary Fig. 3). Actually, $14.3 \%$ of the pregnant women took multivitamin 
supplement containing iodine during pregnancy. About 90.7\% of the pregnant women used iodized salt in this cohort. Macrosomia (weight $>4000 \mathrm{~g}$ ) accounted for $4 \%$ of all infants (Table 1). More than $70 \%$ of pregnant women in this study were in the second trimester and had higher iodine levels than those in the first and third trimesters. Multivitamin supplement with iodine and frequent milk intake was associated with higher UIC $(P<0.0001)$. Iodized salt intake significantly elevated UIC compared with people who never eat iodized salt $(P<0.0001)$. Frequent seafood intake was associated with higher UIC, although not statistically significant (Supplementary Table 1).

\section{Maternal diet and neonatal characteristics}

Multivitamin supplements containing iodine, iodized salt intake, and frequent consumption of seafood, milk and yogurt was significantly associated with a heavier birthweight. Iodized salt intake was significantly related to the larger head circumference of the newborns. Iodized salt and frequent seafood intake were associated with longer femur length (Table 2). No significant correlation was observed between neonatal characteristics and maternal age and pre-pregnancy BMI.

\section{Risk factors for exceeding adequate iodine intake}

Clinical factors, such as multivitamin supplement with iodine and dietary intake are considered risk factors for iodine excess. In this study, more than adequate iodine (UIC $\geq 250 \mu \mathrm{g} / \mathrm{L}$ ) was used as response variable, and risk factors as explanatory variables in the multivariate logistic regression. We found that multivitamin (containing iodine) supplements and milk intake were risk factors after additional adjustment for other risk factors (Supplementary Table 2).

\section{Maternal iodine status and macrosomia risk}

Significant difference was found in birthweight among different UIC groups. Compared with the pregnant women in the UIC $<250 \mu \mathrm{g} / \mathrm{L}$ group, the birthweight in the UIC $\geq 250 \mu \mathrm{g} / \mathrm{L}$ group was heavier $(P<0.05)$. The femur length in the UIC of $250-499 \mu \mathrm{g} / \mathrm{L}$ group was longer than that $<150 \mu \mathrm{g} / \mathrm{L}$ group $(P<0.05)$. In the group with UIC $<150 \mu \mathrm{g} / \mathrm{L}$, the infant head circumference was smaller than other groups $(P<0.05) \quad$ (Supplementary Table 3). Multivariate logistic regression was used to evaluate maternal UIC and macrosomia risk. More than adequate (UIC $\geq 250 \mu \mathrm{g} / \mathrm{L}$ ) and iodine excess (UIC $\geq$ $500 \mu \mathrm{g} / \mathrm{L}$ ) during pregnancy acted as risk factors for macrosomia regardless of gestational weeks (Table 3) while iodine deficiency was not a risk factor for macrosomia (Supplementary Table 4).

\section{Monitor models for guide iodine supplement to prevent more than adequate iodine intake}

Multinomial logistic regression models based on multiple variables were built to assess the risk of above adequate iodine intake (Fig. 1). Conventional risk factors: multivitamin supplement with iodine, iodized salt and dietary habits were incorporated into the model. The feature importance of variables in the models for different trimesters were shown in Supplementary Fig. 4. The area under the ROC curve (AUC) of the monitoring models of the first trimester of pregnancy (pregnancy stage I), second trimester (pregnancy stage II) and third trimester (pregnancy stage III) were $0.70,0.73$ and 0.77 , respectively in the test set $(n=400)$ (Fig. 1) and AUC of models of the three trimesters of pregnancy in the train set $(n=470)$ were $0.72,0.73$ and 0.81 shown in Supplementary Fig. 5. Based on the models, the adjustable parameters like adjust edible salt or multivitamin supplements to non-iodized ones or reducing iodinerich food consumption can be recommended to pregnant women to prevent more than adequate iodine intake. Besides, it is helpful to better understand the iodine nutrition status of local pregnant women and timely adjust the iodine intake strategy (Table 4).

\section{Discussion}

Maternal iodine status is important for the growth and development of fetus. Severe iodine deficiency or iodine excess is related to adverse fetus outcomes [16]. The World Health Organization (WHO) currently recommends using UIC from spot urine samples to describe the iodine status of a population. Despite the WHO recommendation, the sampling is convenient. 24-h urinary iodine excretion (UIE) was used as a reference standard for estimation of iodine intake. In our follow-up of more than a year, urine volume was affected by climate and drinking water, and fluctuated greatly. Therefore, we consider the operability and convenience of collection, and used spot urine to evaluate iodine nutritional status. The WHO recommends that the median UIC for pregnant women is $150-249 \mu \mathrm{g} / \mathrm{L}$ [17]. This study showed that the median UIC for pregnant women was $172 \pm$ $135 \mu \mathrm{g} / \mathrm{L}$ (sufficient by WHO criteria) which was similar compared with previous study which included 2087 pregnant women in Wuhan City, another city in Hubei Province and found that the average UIC was $178 \mu \mathrm{g} / \mathrm{L}$. Universal salt iodization is the first-line strategy for the elimination of severe iodine deficiency. After the implementation of a USI policy [18], China has nearly wiped out IDD in the past two decades. In addition, increasing dietary iodine intake is an important manner to prevent iodine deficiency. Authorities recommend that pregnant women should supplement $150 \mathrm{mg}$ iodine daily to achieve a total daily iodine intake of $250 \mathrm{mg}$ [19]. Here, 
Table 1 Baseline characteristics for total participants

\begin{tabular}{l} 
Characteristics \\
\hline Total pregnancy women \\
Age, years \\
$0-25$ \\
$26 \sim 35$ \\
$>35$ \\
Gestational week \\
first trimester \\
second trimester \\
third trimester \\
Pre-pregnancy BMl, $\mathrm{kg} / \mathrm{m}^{2}$ \\
$<18.5$ \\
$18.5 \sim 24$ \\
$24.1 \sim 28.9$ \\
$\geq 29$
\end{tabular}

n (\%)

870

$181(20.8)$

$643(73.9)$

$46(5.3)$

$178(20.5)$

$628(72.2)$

$64(7.4)$

$63(7.2)$

$569(65.4)$

$175(20.1)$

$63(7.2)$

Multivitamin with iodine

Yes

$124(14.3)$

$746(85.7)$

lodized salt

Yes

789 (90.7)

$81(9.3)$

Sea food (fish, crab, shrimp)

Frequent

$135(15.5)$

Occasional

$667(76.7)$

None

Cow' milk

Frequent

Occasional

None

Yogurt

Frequent

Occasional

None

$68(7.8)$

$412(47.4)$

397 (45.6)

$61(7.0)$

144 (16.6)

$521(59.9)$

205 (23.6)

Egg

Frequent

347 (39.9)

$478(54.9)$

$45(5.2)$

870

Total infants

Infants gender

Male

468 (53.79)

Female

402 (46.21)

Infants weight (g)

$<4000$

$\geq 4000$

Infants length $(\mathrm{cm})$
$28.4 \pm 4.0$

Mean \pm SD

$16.6 \pm 6.9$

$22.7 \pm 3.7$ 
Table 1 Baseline characteristics for total participants (Continued)

\begin{tabular}{lrr}
\hline Characteristics & $\mathbf{n ~ ( \% )}$ & Mean \pm SD \\
\hline Femur length $(\mathrm{cm})$ & & $7.3 \pm 0.3$ \\
Head circumference $(\mathrm{cm})$ & $33.6 \pm 1.3$ \\
\hline SD standard deviation & & \\
BMI body mass index & & \\
UIC urinary iodine concentration &
\end{tabular}

we found significant associations between iodine contained supplements and UIC though only $14.3 \%$ of pregnant women in this cohort took iodine contained supplements. What's more, it comes from multivitamin (containing iodine) supplements. A positive significant correlation between UIC and more frequent milk consumption was found which was consistent with previous studies [20-23]. Seafood is still the main food source of iodine and positively correlated with UIC in vivo. A study conducted in Korea showed a significant correlation between dietary iodine in seaweed and UIC [24].
However, in central China, seafood is not the main diet for residents, and only $15.5 \%$ of the subjects in this study consumed seafood frequently, so there was no significant correlation between seafood consumption and UIC. In this cohort, $90.7 \%$ of pregnant women used iodized salt, which was associated with higher UIC compared to pregnant women not taking iodized salt. According to the urinary iodine level of Chinese residents, the amount of iodine in iodized salt is always changing. In 2011, the Ministry of Health of the People's Republic of China issued the "iodine content of edible salt", which stipulates

Table 2 Correlation between neonatal characteristics and maternal clinical factors

\begin{tabular}{|c|c|c|c|c|c|c|c|c|}
\hline & Birth height $(\mathrm{cm})$ & $P$ & Birth weight (g) & $P$ & Femur length $(\mathrm{cm})$ & $P$ & Head circumference $(\mathrm{cm})$ & $P$ \\
\hline \multicolumn{9}{|c|}{ Multivitamin with iodine } \\
\hline Yes & $50(49,51)$ & 0.231 & $3420(3298,3600)$ & $0.001^{*}$ & $7.3(7.1,7.5)$ & 0.996 & $33.5(33.0,34.5)$ & 0.455 \\
\hline No & $50(49,51)$ & & $3383(3200,3550)$ & & $7.3(7.1,7.5)$ & & $33.5(33.0,34.2)$ & \\
\hline \multicolumn{9}{|l|}{ Drinker } \\
\hline Yes & $50(49,51)$ & 0.705 & $3440(3250,3600)$ & 0.078 & $7.3(7.1,7.4)$ & 0.292 & $33.5(33.0,34.4)$ & 0.771 \\
\hline No & $50(49,51)$ & & $3400(3200,3550)$ & & $7.3(7.1,7.5)$ & & $33.5(33.0,34.2)$ & \\
\hline \multicolumn{9}{|l|}{ Sea food } \\
\hline Frequent & $50(49,51)$ & 0.964 & $3450(3200,3600)$ & $0.015^{*}$ & $7.4(7.2,7.6)$ & $0.034^{*}$ & $33.5(33.0,34.2)$ & 0.290 \\
\hline Occasional & $50(49,51)$ & & $3400(3200,3520)$ & & $7.3(7.1,7.5)$ & & $33.6(33.1,34.3)$ & \\
\hline None & $50(49,51)$ & & $3420(3250,3600)$ & & $7.3(7.0,7.5)$ & & $33.5(32.8,34.1)$ & \\
\hline \multicolumn{9}{|l|}{ Egg } \\
\hline Frequent & $50(49,51)$ & 0.440 & $3400(3200,3560)$ & 0.550 & $7.3(7.1,7.5)$ & 0.100 & $33.5(33.0,34.4)$ & 0.536 \\
\hline Occasional & $50(49,51)$ & & $3400(3200,3543)$ & & $7.3(7.1,7.5)$ & & $33.5(33.0,34.2)$ & \\
\hline None & $50(50,51)$ & & $3450(3300,3560)$ & & $7.5(7.2,7.6)$ & & $33.7(33.2,34.2)$ & \\
\hline \multicolumn{9}{|l|}{ Cow' milk } \\
\hline Frequent & $50(49,51)$ & 0.365 & $3420(3208,3600)$ & $<0.001^{*}$ & $7.3(7.1,7.5)$ & 0.594 & $33.6(33.1,34.3)$ & 0.094 \\
\hline Occasional & $50(49,51)$ & & $3400(3200,3520)$ & & $7.3(7.1,7.5)$ & & $33.5(33.0,34.2)$ & \\
\hline None & $50(49,51)$ & & $3300(3150,3500)$ & & $7.2(7.0,7.5)$ & & $33.5(33.0,33.9)$ & \\
\hline \multicolumn{9}{|l|}{ Yogurt } \\
\hline Frequent & $50(50,51)$ & 0.061 & $3450(3250,3625)$ & $0.037^{*}$ & $7.3(7.2,7.5)$ & 0.710 & $33.5(33.0,34.1)$ & 0.521 \\
\hline Occasional & $50(49,51)$ & & $3400(3200,3520)$ & & $7.3(7.1,7.5)$ & & $33.5(33.0,34.2)$ & \\
\hline None & $50(49,51)$ & & $3320(3200,3550)$ & & $7.3(7.1,7.5)$ & & $33.6(33.2,34.3)$ & \\
\hline \multicolumn{9}{|l|}{ lodized salt } \\
\hline Yes & $50(49,51)$ & 0.868 & $3400(3200,3560)$ & $0.002^{*}$ & $7.3(7.1,7.5)$ & $<0.001^{*}$ & $33.6(33.0,34.3)$ & $<0.001^{*}$ \\
\hline No & $50(49,51)$ & & $3340(3152,3450)$ & & $7.2(7.0,7.3)$ & & $33.4(32.9,33.6)$ & \\
\hline
\end{tabular}

Data presented as mean (interquartile range, IQR)

*Statistically significant difference set at $P<0.05$, Kruskal-Wallis $\mathrm{H}$ test 
Table 3 Multivariate logistic regression analysis on macrosomia risk

\begin{tabular}{lll}
\hline UIC $(\boldsymbol{\mu g} / \mathrm{L})$ & $\begin{array}{l}\text { Macrosomia } \\
\text { OR }(\mathbf{9 5 \%} \text { CI })\end{array}$ & $\boldsymbol{P}$-value \\
\hline $0-149$ & Ref & \\
$150-249$ & $1.40(0.53,3.68)$ & 0.496 \\
$250-499$ & $2.94(1.25,6.94)$ & $0.014^{*}$ \\
$\geq 500$ & $2.66(1.51,7.78)$ & $0.043^{*}$ \\
\hline
\end{tabular}

Data presented as odds ratio (OR) (95\% confidence interval (CI))

*Statistically significant difference set at $P<0.05$

that the average level of iodine content in edible salt can be divided into $20 \mathrm{mg} / \mathrm{kg}, 25 \mathrm{mg} / \mathrm{kg}$ and $30 \mathrm{mg} / \mathrm{kg}$. One or two kinds of iodized salt can be selected according to the iodine nutrition level of people in the province in China. Xiangyang and Pingdingshan municipal government both chose $25 \mathrm{mg} / \mathrm{kg}$ standard. We confirmed the amount of iodization through the salt package provided by pregnant women. But still not sure if this dose is appropriate for each individual. Clinical factors, such as multivitamin supplements containing iodine and dietary intake are considered risk factors for iodine excess. We recalculated odds ratio (OR) value in multivariable logistic regression model, and found that two variables, multivitamin supplements with iodine and frequent milk consumption, with OR greater than 1 and $\mathrm{P}$ less than 0.05 , were regarded as risk factors for more than adequate iodine (UIC $\geq 250 \mu \mathrm{g} / \mathrm{L}$ ) (Supplementary Table 2). However, they are not independent risk factor. However, the increase of thyroid diseases related to excessive iodized salt has become a new public health problem [2527]. The harm of iodine excess to pregnant women and fetus, the recommended safe dose of iodine intake is controversial and, especially for women with mild to moderate iodine deficiency. Routine monitoring is necessary to guarantee adequate iodine status. Therefore, the present study aimed to assess iodine status in central China and explore the effect of maternal UIC on neonatal outcomes. Because the best indicator to evaluate iodine status is still controversial.

Infants with maternal UIC below $150 \mu \mathrm{g} / \mathrm{L}$ had lower birthweight, shorter femur length and smaller head circumference than those with maternal UIC between 250 and $449 \mu \mathrm{g} / \mathrm{L}$ in our study. Otherwise, this study demonstrated that neonatal features were associated with dairy food and supplements. Iodine supplement, seafood, milk, yogurt intake and iodized salt consumption were positively associated with birthweight. This finding above was consistent with previous review which suggested the associations between iodized salt consumption and the increase of birthweight [28, 29]. Significant difference was also found in birthweight among different UIC groups (the birthweight in the UIC $\geq 250 \mu \mathrm{g} / \mathrm{L}$ group was heavier). More macrosomia occurred in pregnant women with more than adequate iodine than in those with iodine deficiency $(52.8 \%$ vs. $16.7 \%, P=0.001)$. Then multivariate logistic regression analysis was used to evaluate whether UIC was the risk factor for macrosomia risk. The result showed that more than adequate $(\mathrm{UIC} \geq 250 \mu \mathrm{g} / \mathrm{L}$ ) and iodine excess (UIC $\geq 500 \mu \mathrm{g} / \mathrm{L}$ ) during pregnancy acted as risk factors for macrosomia. Therefore, for pregnant women in central China who generally ate iodized salt, they need to take

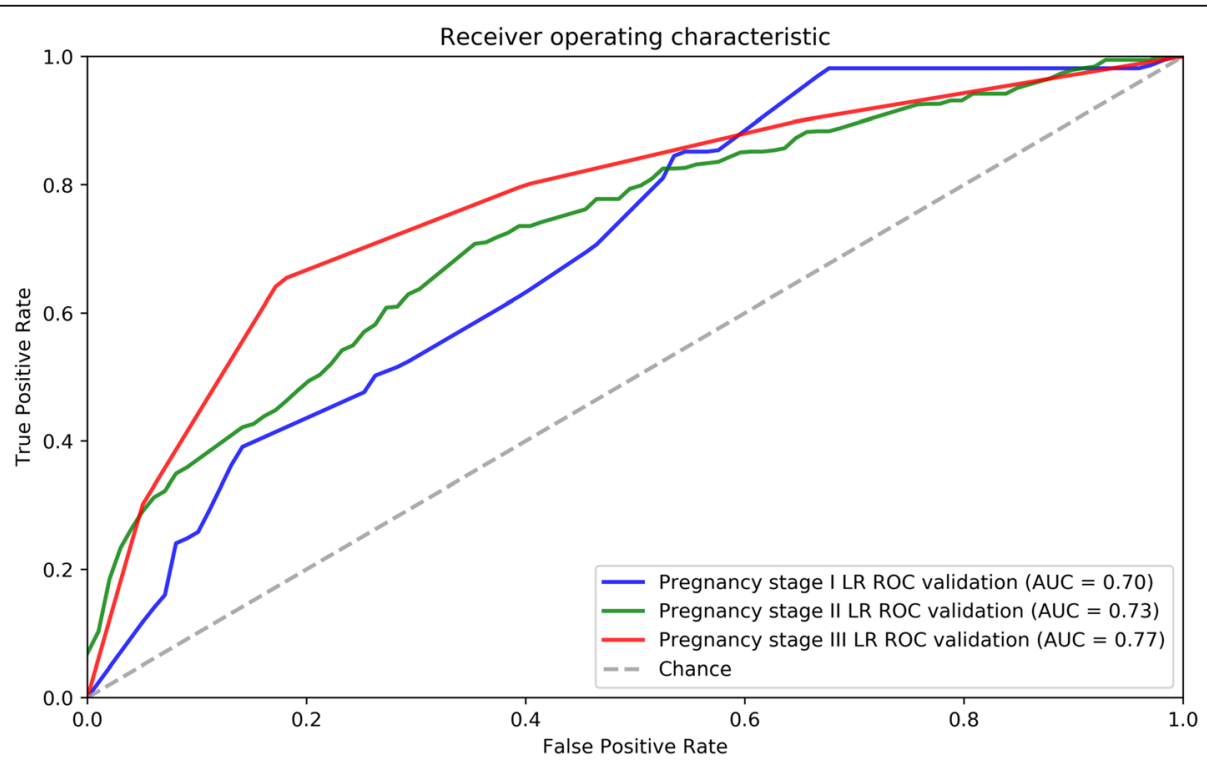

Fig. 1 Logistic regression models of monitoring UIC among pregnancy women of the three trimesters. A The AUC of the model of the first trimester was 0.70. B The AUC of the model of the second trimester was 0.73. C The AUC of the model of the third trimester was 0.77 
Table 4 Clinical application of the models for predicting the risk of more than adequate iodine intake

\begin{tabular}{|c|c|c|c|}
\hline Trimester & Score & $\begin{array}{l}\text { More than adequate } \\
\text { risk }\end{array}$ & Clinical recommendations \\
\hline \multirow[t]{3}{*}{ I } & $\begin{array}{l}0.53 \sim \\
1.00\end{array}$ & Medium & Replace iodized salt or reduce iodine-rich food intake according to the model \\
\hline & $0.22 \sim$ & Medium Low & Reduce iodine-rich food intake according to the model \\
\hline & $0.00 \sim$ & Low & Monitor UIC \\
\hline \multirow[t]{3}{*}{$\|$} & $\begin{array}{l}0.56 \sim \\
1.00\end{array}$ & Medium High & $\begin{array}{l}\text { Do not eat iodine contained supplements and reduce iodine-rich food intake according to the } \\
\text { model }\end{array}$ \\
\hline & $\begin{array}{l}0.20 \sim \\
0.56\end{array}$ & Medium Low & Reduce iodine-rich food intake according to the model \\
\hline & $\begin{array}{l}0.00 \sim \\
0.20\end{array}$ & Low & Monitor UIC \\
\hline \multirow[t]{2}{*}{ III } & $\begin{array}{l}0.58 \sim \\
1.00\end{array}$ & Medium High & Don't eat iodine contained supplements \\
\hline & $\begin{array}{l}0.00 \sim \\
0.58\end{array}$ & Low & Monitor UIC \\
\hline
\end{tabular}

iodine-containing multivitamin carefully. No correlation between UIC during the third trimester and first trimester of pregnancy and macrosomia was observed. High thyroid-stimulating hormone (TSH) $(\mathrm{TSH}>4.94 \mathrm{nIU} / \mathrm{l})$ or low free thyroxine (FT4) $(\mathrm{FT} 4<$ 9.01 pmol / L) had no adverse effect on macrosomia. Iodine deficiency was also not a risk factor for macrosomia (Supplementary Table 4). To monitor iodine status to prevent the risk of more than adequate iodine intake in pregnant women with normal UIC at present, thus avoiding the risk of adverse pregnancy outcomes such as macrosomia, the clinical model is established to monitor the iodine nutritional status of pregnant women in the central China to avoid more than adequate iodine intake, thus reducing the risk of having a macrosomia. Because the random urine iodine test can only represent the iodine level of this time and cannot fully reflect the iodine status in the body, nor can it show that iodized salt or iodine-rich diet intake is suitable, so how to predict the high-risk group of pregnant women with more than adequate iodine through multiple variables is meaningful, especial for pregnant women in central China who generally eat iodized salt, they need to take iodinecontaining multivitamin or food carefully. In the machine learning framework, in brief, this is an online model. The model is based on the data of 870 pregnant women. The intake of iodized salt and the frequency of milk and other iodine-rich diet was taken as variables and input into the model, which have different important values (Supplementary Fig. 4). Then they will get a prediction score, which indicates the iodine nutritional status of the pregnant women, especially the pregnant women whose UIC showed that iodine was adequate can avoid iodine excess via adjustment measures given by the model. We believe that the combination of multiple risk factors is more effective in predicting risk than single indicator such as random spot urinary iodine or 24-h UIE. Then clinical guidance such as replacing iodine contained salt or supplements to non-iodine ones or reducing iodine-rich food can be output according to the risk range, and the frequency of reduction can be output, thus helping clinicians to provide prevention for more than adequate iodine intake in the pregnant women in different pregnant time. For pregnant women whose UIC has exceeded the adequate range, they can cooperate with the UIC value to evaluate the iodine-rich diet of pregnant women and give adjustment measures. The sample was relatively small in this study, thus a large population in the further studies will be analyzed to verify the role of the monitor models in clinical practice.

\section{Conclusions}

Iodine is currently considered to be sufficient in the central China. But it is necessary to publicize the harm of iodine-excess, and its potential risk of macrosomia. The clinical model of each trimester of pregnancy was established to minimize the risk of more than adequate iodine intake, thus reducing the risk of adverse pregnancy outcomes. In addition, it is helpful to better understand the iodine nutrition status of local pregnant women and timely adjust the iodine intake strategy.

\section{Abbreviations}

UIC: Urinary iodine concentration; TH: Thyroid hormones; WHO: World Health Organization; USI: Universal salt iodization; IDD: Iodine deficiency disease: BMI: Body mass index; SD: Standard deviation; ROC: Receiver operating 
characteristic; AUC: Area under the curve; TSH: thyroid-stimulating hormone; FT4: Low free thyroxineOROdds ratio; Cl: Confidence interval

\section{Supplementary Information}

The online version contains supplementary material available at https://doi. org/10.1186/s12884-021-03936-w.

\section{Additional file 1}

\section{Acknowledgments}

We are particularly grateful to all the participants in this research. We thank for the efforts of Xiangyang No.1 People's Hospital and Pingdingshan No.1 People's Hospital.

\section{Authors' contributions}

JJD and JD were responsible for the study design; WYX wrote the main manuscript; LYW, SYL, XJZ, QHZ, CLL and JRZ were responsible for collection of samples and questionnaires; WLY did data analysis for the Fig. 1 and tables. All authors reviewed and approved the manuscript.

\section{Funding}

No funding

\section{Availability of data and materials}

The datasets used and/or analysed during the current study available from the corresponding author on reasonable request.

\section{Declarations}

Ethics approval and consent to participate

The study has been performed in accordance with the Declaration of Helsinki and was approved by the Research Ethics Committee (201702170b) of Hubei Medical College. Written informed consent was obtained from participants.

\section{Consent for publication}

Not applicable.

\section{Competing interests}

Authors state no conflict of interest.

\begin{abstract}
Author details
'Department of Gynaecology and Obstetrics, Xiangyang No.1 People's Hospital, Hubei University of Medcine, Xiangyang 441000, China. 2Department of Obstetrics, Pingdingshan No.1 People's Hospital, Pingdingshan 467000, China. ${ }^{3}$ Biotecan Medical Diagnostics Co., Ltd, Zhangjiang Center for Translational Medicine, Shanghai 201204, China.

${ }^{4}$ Singlera Genomics (Shanghai) Ltd, Shanghai 201318, China.
\end{abstract}

Received: 2 March 2021 Accepted: 4 June 2021

Published online: 28 June 2021

\section{References}

1. de Escobar GM, Obregon MJ, del Rey FE. Maternal thyroid hormones early in pregnancy and fetal brain development. Best Pract Res Clin Endocrinol Metab. 2004;18(2):225-48. https://doi.org/10.1016/j.beem.2004.03.012.

2. Glinoer D. The regulation of thyroid function during normal pregnancy: importance of the iodine nutrition status. Best Pract Res Clin Endocrinol Metab. 2004;18(2):133-52. https://doi.org/10.1016/j.beem.2004.03.001.

3. Qian M, Wang D, Watkins WE, Gebski V, Yan YQ, Li M, et al. The effects of iodine on intelligence in children: a meta-analysis of studies conducted in China. Asia Pac J Clin Nutr. 2005;14(1):32-42

4. Abalovich M, Gutierrez S, Alcaraz G, Maccallini G, Garcia A, Levalle O. Overt and subclinical hypothyroidism complicating pregnancy. Thyroid. 2002;12(1): 63-8. https://doi.org/10.1089/105072502753451986.

5. Allan WC, Haddow JE, Palomaki GE, Williams JR, Mitchell ML, Hermos RJ, et al. Maternal thyroid deficiency and pregnancy complications: implications for population screening. J Med Screen. 2000;7(3):127-30. https://doi.org/1 0.1136/jms.7.3.127
6. Das SC, Mohammed AZ, Al-Hassan S, Otokwula AA, Isichei UP. Effect of environmental iodine deficiency (EID) on foetal growth in Nigeria. Indian J Med Res. 2006;124(5):535-44.

7. Xiao Y, Sun H, Li C, Li Y, Peng S, Fan C, et al. Effect of iodine nutrition on pregnancy outcomes in an iodine-sufficient area in China. Biol Trace Elem Res. 2018;182(2):231-7. https://doi.org/10.1007/s12011-017-1101-4.

8. Nicola JP, Basquin C, Portulano C, Reyna-Neyra A, Paroder M, Carrasco N. The $\mathrm{Na}+/$ - symporter mediates active iodide uptake in the intestine. Am J Physiol Cell Physiol. 2009;296(4):C654-62. https:/doi.org/10.1152/ajpcell.00509.2008.

9. Kusic Z, Jukic T, Rogan SA, Juresa V, Dabelic N, Stanicic J, et al. Current status of iodine intake in Croatia--the results of 2009 survey. Coll Antropol. 2012;36(1):123-8.

10. Yang L, Li M, Liu X, Wu M, Zhang J, Zhao L, et al. Evaluation of iodine nutritional status among pregnant women in China. Thyroid. 2020;30(3): 443-50. https://doi.org/10.1089/thy.2019.0001.

11. Chen R, Li Q, Cui W, Wang X, Gao Q, Zhong C, et al. Maternal iodine insufficiency and excess are associated with adverse effects on fetal growth: a prospective cohort study in Wuhan, China. J Nutr. 2018;148(11):1814-20. https://doi.org/10.1093/jn/nxy182.

12. Yang J, Liu Y, Liu H, Zheng H, Li X, Zhu L, et al. Associations of maternal iodine status and thyroid function with adverse pregnancy outcomes in Henan Province of China. J Trace Elem Med Biol. 2018;47:104-10. https://doi. org/10.1016/j.jtemb.2018.01.013.

13. Yang J, Yan JZ, Li XF, Zheng HM, Zhu L, Liu Y, et al. lodine status of vulnerable populations in Henan Province of China three years after the implementation of new iodized salt standard. Zhonghua Yu Fang Yi Xue Za Zhi. 2019;53(11):1173-5.

14. Lee JH, Ji OJ, Song MJ, Park HD, Kim HK, Kim SW, et al. Determination of urinary iodine concentration by inductively coupled plasma-mass spectrometry in thyroid cancer patients on low-iodine diet. Korean J Lab Med. 2010;30(4):351-6. https://doi.org/10.3343/kjlm.2010.30.4.351.

15. WHO. Urinary iodine concentrations for determining iodine status in populations. Vitamin and Mineral Nutrition Information System. Geneva: World Health Organization; 2013. https://www.who.int/publications/i/item/ WHO-NMH-NHD-EPG-13.1.

16. Zimmermann MB. The effects of iodine deficiency in pregnancy and infancy. Paediatr Perinat Epidemiol. 2012;26(Suppl 1):108-17. https://doi. org/10.1111/j.1365-3016.2012.01275.x.

17. WHO. ICCIDD \& UNICEF (2007) Assessment of the lodine Deficiency Disorders and Monitoring their Elimination, 3rd ed. 2007.

18. Cui SL, Liu P, Su XH, Liu SJ. Surveys in areas of high risk of iodine deficiency and iodine excess in China, 2012-2014: current status and examination of the relationship between urinary iodine concentration and goiter prevalence in children aged 8-10 years. Biomed Environ Sci. 2017;30(2):8896. https://doi.org/10.3967/bes2017.012.

19. Alexander EK, Pearce EN, Brent GA, Brown RS, Chen H, Dosiou C, et al. 2017 guidelines of the American Thyroid Association for the diagnosis and Management of Thyroid Disease during Pregnancy and the postpartum. Thyroid. 2017:27(3):315-89. https://doi.org/10.1089/thy.2016.0457.

20. Bath SC, Furmidge-Owen VL, Redman CW, Rayman MP. Gestational changes in iodine status in a cohort study of pregnant women from the United Kingdom: season as an effect modifier. Am J Clin Nutr. 2015;101(6):1180-7. https://doi.org/10.3945/ajen.114.105536.

21. Bath SC, Walter A, Taylor A, Wright J, Rayman MP. lodine deficiency in pregnant women living in the south east of the UK: the influence of diet and nutritional supplements on iodine status. Br J Nutr. 2014;111(9):162231. https://doi.org/10.1017/S0007114513004030.

22. Mian C, Vitaliano P, Pozza D, Barollo S, Pitton M, Callegari G, et al. lodine status in pregnancy: role of dietary habits and geographical origin. Clin Endocrinol. 2009;70(5):776-80. https://doi.org/10.1111/j.13 65-2265.2008.03416.x

23. Vanderpump MP, Lazarus JH, Smyth PP, Laurberg P, Holder RL, Boelaert K, et al. lodine status of UK schoolgirls: a cross-sectional survey. Lancet. 2011; 377(9782):2007-12. https://doi.org/10.1016/S0140-6736(11)60693-4.

24. Kim JY, Moon SJ, Kim KR, Sohn CY, Oh JJ. Dietary iodine intake and urinary iodine excretion in normal Korean adults. Yonsei Med J. 1998;39(4):355-62. https://doi.org/10.3349/ymj.1998.39.4.355.

25. Bastemir M, Emral R, Erdogan G, Gullu S. High prevalence of thyroid dysfunction and autoimmune thyroiditis in adolescents after elimination of iodine deficiency in the eastern Black Sea region of Turkey. Thyroid. 2006: 16(12):1265-71. https://doi.org/10.1089/thy.2006.16.1265. 
26. Pedersen IB, Laurberg P, Knudsen N, Jorgensen T, Perrild H, Ovesen L, et al. An increased incidence of overt hypothyroidism after iodine fortification of salt in Denmark: a prospective population study. J Clin Endocrinol Metab. 2007;92(8):3122-7. https://doi.org/10.1210/jc.2007-0732.

27. Teng W, Shan Z, Teng X, Guan H, Li Y, Teng D, et al. Effect of iodine intake on thyroid diseases in China. N Engl J Med. 2006;354(26):2783-93. https:// doi.org/10.1056/NEJMoa054022

28. Mason JB, Deitchler M, Gilman A, Gillenwater K, Shuaib M, Hotchkiss D, et al. lodine fortification is related to increased weight-for-age and birthweight in children in Asia. Food Nutr Bull. 2002;23(3):292-308. https://doi.org/10.11 $77 / 156482650202300309$

29. Nazeri P, Shab-Bidar S, Pearce EN, Shariat M. Do maternal urinary iodine concentration or thyroid hormones within the normal range during pregnancy affect growth parameters at birth? A systematic review and meta-analysis. Nutr Rev. 2020;78(9):747-63. https://doi.org/10.1093/nutrit/ nuz105.

\section{Publisher's Note}

Springer Nature remains neutral with regard to jurisdictional claims in published maps and institutional affiliations.

Ready to submit your research? Choose BMC and benefit from:

- fast, convenient online submission

- thorough peer review by experienced researchers in your field

- rapid publication on acceptance

- support for research data, including large and complex data types

- gold Open Access which fosters wider collaboration and increased citations

- maximum visibility for your research: over $100 \mathrm{M}$ website views per year

At BMC, research is always in progress.

Learn more biomedcentral.com/submissions 